\title{
Actinomicosis Abdominal Infantil Reporte de un Caso Clínico
}

Camen Albornoz Valel ${ }_{2}$, Luis Zuror ${ }^{2}$, Marianela Caro. ${ }^{3}$

\begin{abstract}
A child ages 3 years suffered an abmominal truuma with a very litle periuration at the small intestine.

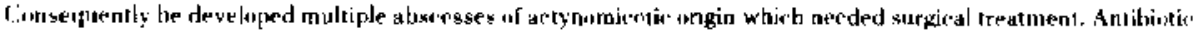
were assinclated and used fir a ling periinl of lime.

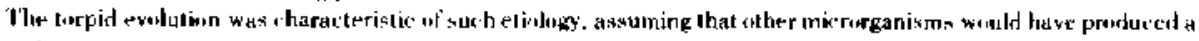
'quirurgic emergenty.
\end{abstract}

La Actinomicusis es una enfermedad crónica supurada producida por el Actinomyces israelii. germen descrito por primera vez por Israel en 1885 . El Actinomyces (Act) es un bacilo Gram positivo ramificado, anaerobio, que se encuentra formando parte de la flora bucal y gastrointestinal, comenzando a colonizar desde los dos meses de edad" (Figura 1 ).

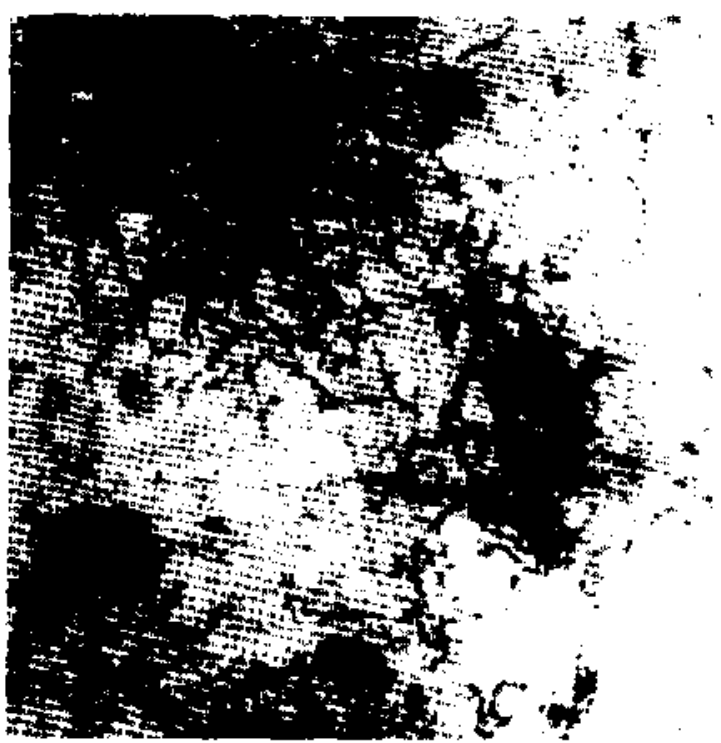

Figura 1. Actinomyces israelii, bacilo Gram pusitivo ramificado.

El génern Act. tiene cinco especies: Act, israelii, Act. bovis, Act. odontolyticus, Act, naeslendii. Act. viscosus, siendo el prirnero el responsable de esta entidad clínica en el hombre.

\footnotetext{
lMédico becario, Hospital Regional Valdivia.

2Tecniilugo médico, Institute de Microbiologia, Unjversidad Austrał de Chile.

3Médicos, Servicio de Cïrugía Infantil. Hospital Regianal Vuldivia.
}

Se describen tres formas de presentación: cervicofacial, torácica y abdominal cuya frecuencia es variable de una serie a otra.

Frecuencia de las formas clínicas de Actinomicosis Autores

\begin{tabular}{llcc} 
Ubicacion & $\begin{array}{c}\text { Weese } \\
\text { (57 casos) }\end{array}$ & $\begin{array}{c}\text { Harvey } \\
(37 \text { casos) }\end{array}$ & $\begin{array}{c}\text { Brown } \\
\text { (181 casos) }\end{array}$ \\
\hline Cervicofacial & $50 \%$ & $24 \%$ & $35.3 \%$ \\
Toricico & $17,6 \%$ & $13 \%$ & $33.5 \%$ \\
Abdominal & $22,8 \%$ & $63 \%$ & $32.3 \%$ \\
\hline
\end{tabular}

En los niños está afección es rara, siendo muy pocos los casos encontrados en la literatura mundial, lo que motivó la comunicación del hallazgo de un caso de Actinomicosis en un nir̃o hospitalizado en el Servicio de Pediatria, Hospital Regional de Valdivia.

\section{CASO CLINICO}

J. S. H.. sexo masculino, 3 años procedente de Choshuenco, Panguipulli. Ingresado al Servicio de Pediatria, Hospital Regional de Valdivia el 5 de septiembre de 1978, con el antecedente de haber sufrido dos diss antes, un traumatio mo abodominal, cuyo impacto mayor se localizí en región inguimopubiana izquierda.

Al examen general se constataba un nin̄o decaído, quejumbruso, abdomen distendido, no depresible, sensible en flanco izquierdo e hipogastrio, resistencia muscular y ruidos intestinales disminuidos. La radiografía de abdomen simple de urgencia demostró una gran dilatación de asas de intestino delgadu, con pasaje de gas a niveles más bajos, lo cual descartaba un cutdro quirurgico de urgencia.

Durante los días posteriores evolucionó en forma insatisfactoria llamando la ateneién un lento pero progresivo compromiso del estado general, irritabilidad marcada, deposiciones diarreicas de mal wlor, manteniéndose subfebril. 
Fil hemograma $(6.9 .78$ ) mostri un hematucrito de $35 \%$, leurecitos de $8.800 \times \mathrm{xn}^{3} \mathrm{y}$ desviación a izquiecda de 19 baciliformes. La Velocidad de eritrosedimentación fue de 47 mmx l." hora. (Orina cosi sedimento nomal. hemosultivis y mirloculeivos negativis.

Al cetavo dia presenti dificultad respiraturia, tencuntrándose a la auscultacion foc o de Bronconeumonja biluteral, cue fue arroborada por la radiogratía de tirax. El hemograma de cuntrol mostraba leusocitusis de 19.400 $\mathrm{xmm}^{3}$, y 6 juventles y 13 haviliformes, Sedimentacion de 85 mmx 1, hora. Como la reaceión de aglutinación y cultivas eran persistentemente negativos se decidio ("umenzar trata.

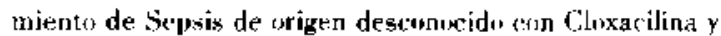
Grontamicina.

Frente a la persistentia clínié, tanto pulmonar como abdoninal. se thicieron puncionus abdominales que resularon en blanco y puxción pulmonar en hemitórax izquierdo, lesde dande se extrajeron 4 tas: de pus fétido, con gránulos como dre semola, coyo Gram directo rovelé Act. israelii, al que fue confirmado por cultivo para anaerulios y las pruebas bioquimicas especificas. El aislamiento se asocici cun Escherichia coli.

Planteandose la prosibilidad de una ruprora intestinal ('omo punto de partida del proceso séptico, se inició tratamiento eun Penicilina sódica $1.000 .000[\mathrm{~J} / \mathrm{Kg}$ y Cloranfenicol 50 mg/Kg, continuanduse esiudio para localizar el focss inicial. La encma baritada reveló un buen pazaje del medio de cuntraste a nivel del colun, sin compresiones extrinsec:as, asas aglutinadas en intestituo delgado de henjabdomen ixquierdo. Fl trinsito intestinal demostró la presencia de una masa retrugástrica que eomprinia su pared porsterior

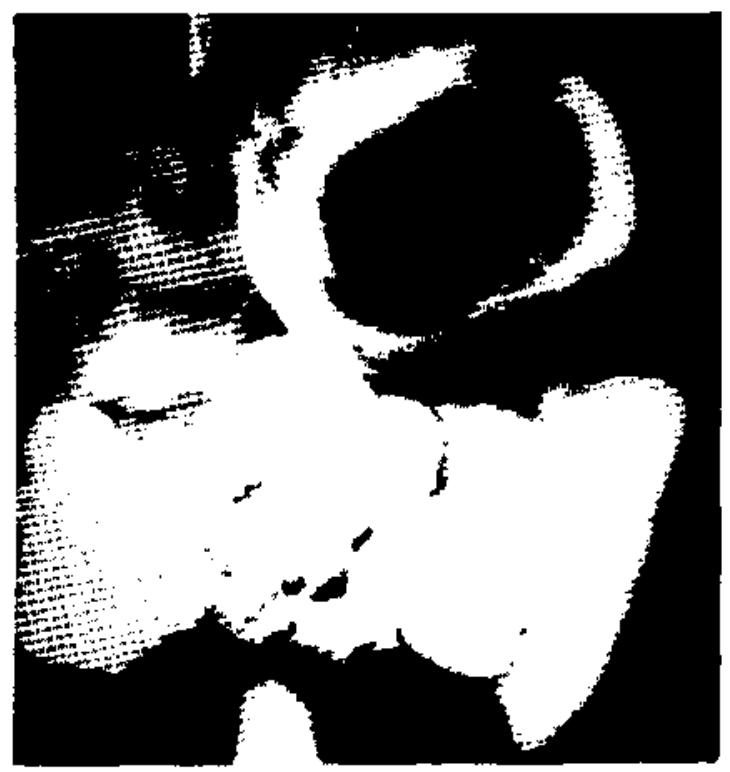

Fipura 2. Tránsite entesinal. gue demestogra desplacamientu del

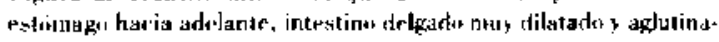

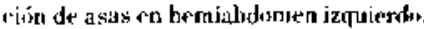

I.a laparulomía exploradira teet ba a 21 dias del ingresos revelí una ruptura puntifiorme do intestibo dedgado en re. giun pariette ijlica izquierda y abscesos: aubfrénico izłuiprdo que rechazaba el estomago havia adelante, en fondo de sal'o prevesical y inesenteriales multiples. Procests inflama1orio de la pared parietricrilica izquierda, subrelusión intesInal meránica lpor procesa inflamatorict). Apéndice indemne. Se practicaron resección intestinal, vaciamiento de abscests y apendirertomia. El esludio histopatoligéco del seymanto resecado destacó una perienteritis aguda y crónica inespecifica abscedada en urganización y los cultivos de las colecutones mevamente fueron pusitivas para Act. isruelii. asociado a Escherichia coli y Racteroides melaninogenirus.

El postoperatorio fue muy tirpida, dándore de alta a lís 57 día de hospitalización en buenas cundiciones generales. a iebril, examents físicos y de laboratorio dentro de límites nurmales, Se cumpleti 10 dias con Clnranfenicol y 25 dias con Penicilina sidica parenteral, manteniéndose la última pur via ural por tres meses al cabo de los cuales el tnenur se encentraba sano.

\section{COMENTARIO}

La Actinomicosis abdominal tiene como punto de partida la perforación intestinal, secundaria a una Apendicitis aguda, Diverticulitis colónica, Ulcera péptica " traumatisno abdominar", el último de $10 \mathrm{~s}$ cuales sería el mecanismo causal del caso que infor. mamos.

El comienzo insidioso con decaimiento, compromiso progresivo del estado general, irritabilidad, annrexia. fiebre y un examen abdominal que aunque siempre estuvo alterado nunca presentó las característic:as de un abdomen agudo constituyen la forma habitual de presentación de este cuadro, motivo por el cual en la gran mayoria de los casos el diagnósticu es de tipo bacteriológico y fo histopatalígieo y no clinico. 3.2 .3 .7 .8

La punción pulmonar hecha en el sexto espacins intercostal izquierdo con alta probabilidad punciono; absceso subfrénico del mismo ladı ya que fuera del Act. se aisló E. coli. Suponemos que como está deserito el cuadro pulmonar fue producido por otro germen aerobio Gram positivo asociado. sensible a Penicilinta. 1. 8. ". 14

Los cultivos hechos en medios corrientes de los abscesos fueron negativos; sin embargo el frotis Gram directo permitió establecer el diagnístico, debido a lo cual pensamos que es recomendable practicar siempre de partida esta técnica a toda muestra clínica para estudio microbiológico, lo cual ahorraria tiempo y orientaria fácilmente tambièn a la siembra en un medio adecuado para confirmar el diagnóstico $y$ a la instauración de una terapia precoz y dirigida. 
El pus encontrado tiene como caracteristica un olor pútrido semejante al de aquellas sepsis wrales de cierta cuantia, que da una fiel aproximación a la sospecha etiológiea.

En relación al estudio histopatológico de la pieza résecada no se encontró el Act., pero la perienteritis en partes aguda y en otras crónica abscedada es un hallazgo común de la Actinomicosis.' Debe considerarse además que el paciente llevaba 13 días con antibiciticos y que la ubicación del germen es focal pudiendo encontrarse solamente en algunos cortes histoligicers.

Por último puede comprobarse una vez más que el tratamiento antibiótico no basta pur sí solo para curar la enfermedad dado que no produjo alivio de la sintomatología. sino por el contrario fue aumentads. Lo útil en estos casos es la asociación de la cirugia para el vaciamiento de las colecciones supuradas y e] tratamiento antibótico prokngado y en altas dosis.' LG. B. 10

\section{REFERENCIAS}

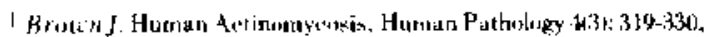
1973.

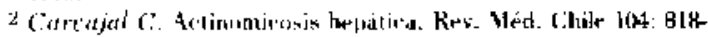
8201. 1976.

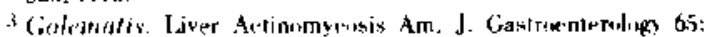
1:18-15l. 1976.

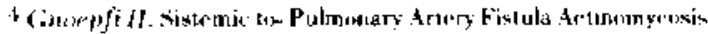
('he 6714): 444-146. 1975.

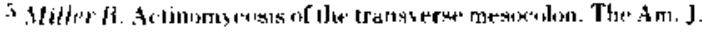
of tiurgery 122: $114-4] 6.19 \% 1$.

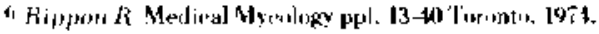

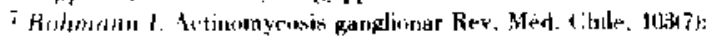
$491+93,1935$.

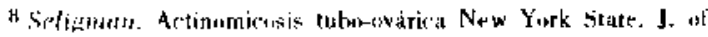
Merl. $76.2 \div: 278280$, 1976.

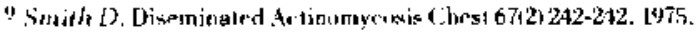

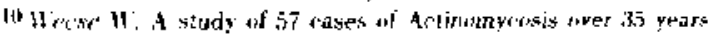
prriud Areh. Int. Med, 1352\% 1562-1568, 1975. 